\title{
Facets for the single node fixed-charge network set with a node set-up variable
}

\author{
Agostinho Agra - Mahdi \\ Doostmohammadi
}

the date of receipt and acceptance should be inserted later

\begin{abstract}
We consider a variant of the well-known Single Node Fixed-Charge Network (SNFCN) set where a set-up variable is associated with the node, indicating whether the node is open or not. This set arises as a relaxation of several practical mixed integer problems. We relate the polyhedral structure of this variant with the polyhedral structure of the SNFCN set. We show that in the presence of the node set-up variable new facet-defining inequalities appear and establish the relation between the new family of inequalities with the flow cover inequalities. For the constant capacitated case we provide a full polyhedral description of the convex hull of the given set.
\end{abstract}

Keywords Mixed integer sets · Valid inequalities · Single node flow set

\section{Introduction}

We consider mixed integer sets of the form

$$
X=\left\{(x, z, y) \in \mathbb{R}_{+}^{n} \times \mathbb{B}^{n} \times \mathbb{B} \mid \sum_{j \in N} x_{j} \leq d y, x_{j} \leq c_{j} z_{j}, j \in N\right\},
$$

where $N=\{1, \ldots, n\}, d>c_{j}>0, \forall j \in N$, and integer.

Set $X$ is much related with the well-known SNFCN set which is the restriction of $X$ to the subspace defined by $y=1$. We also consider set $X^{1}=$ $\{(x, z, y) \in X \mid y=1\}$. The SNFCN set is the projection of $X^{1}$ into the $(x, z)$

Published in Optimization Letters, 8, pp. 1501-1515 2014

A. Agra

Department of Mathematics and CIDMA, University of Aveiro

E-mail: aagra@ua.pt

M. Doostmohammadi

Department of Mathematics and CIDMA, University of Aveiro

E-mail: mahdi@ua.pt 
space. Variable $y$ can be regarded as a set-up variable associated to the node itself. Thus, $y$ indicates whether the capacity of the node is installed or not. So, in the classical SNFCN set the capacity of the node is assumed to be installed. As usual, the binary variables $z_{j}$ are associated with the arcs entering the node and indicating whether the arc is open or not.

The convex hull of $X$ will be denoted by $P$ and the convex hull of the restricted set $X^{1}$ by $P^{1}$.

Set $X$ arises as a relaxation of several mixed integer problems. Next we provide a few examples. In the single-item Lot-sizing with Supplier Selection Problem (LSSP) we are given a set $N$ of suppliers. In each time period one needs to decide lot-sizes and a subset of suppliers to use in order to satisfy the demands while minimizing the costs. For each time period, set $X$ arises as follows: $y$ represents the binary variable indicating whether there is a setup for production or not, $z_{j}$ indicates whether the supplier $j \in N$ is selected or not, $x_{j}$ is the amount supplied by supplier $j, d$ is the production capacity and $c_{j}$ is the supplying capacity of supplier $j$, see [9]. Other examples occur in inventory-routing problems such as the Vendor-Managed Inventory-Routing Problem (see [1]), where, for each time period $t, y$ is a binary variable indicating whether the supplier is visited at time $t$ or not, $z_{j}$ is a binary variable equal to 1 if the retailer $j$ is served at time $t$, and 0 otherwise, $d$ is the capacity of the vehicle, and $c_{j}$ is the maximum inventory level in retailer $j$. In some related problems, $d$ may also represent the inventory capacity and $y$ indicates whether the warehouse is set-up to receive goods or not. Variables $x_{j}$ represent the supplied quantities and variables $z_{j}$ indicate the suppliers selected (as in the LSSP). Other examples can be found where such relaxations occur under particular cases. See, for instance, the Capacitated Location Problem presented in [4] where the binary variable $y$ indicates whether a facility is installed at a given node, $z_{j}$ indicates whether client $j$ is served or not from that node, $x_{j}$ indicates the quantity that the facility sends to client $j \in N$. $d$ represents the facility capacity and $c_{j}$ represents the capacity of the facilityclient link.

Although, as we will show later, valid inequalities derived for $X^{1}$ are, under general conditions, valid for $X$ (and, therefore, can be used to tighten the general mixed integer problems with set-ups on the nodes), a deep study of this particular set is of practical interest, in particular, when the set-up variable may play an important role. Our goal is to provide a better understanding of such sets and explain what can be gained with the explicit inclusion of the set-up variable in the model.

The single node fixed-charge set has been intensively studied in the past, and different variants of the model have been considered. Padberg et al. [7] considered sets of the type

$$
X_{[\triangle]}=\left\{(x, z) \in \mathbb{R}_{+}^{n} \times \mathbb{B}^{n} \mid \sum_{j \in N} x_{j} \triangle d, x_{j} \leq c_{j} z_{j}, j \in N\right\},
$$

where $\triangle \in\{\leq,=, \geq\}$. They introduced the well-known "flow cover" inequalities. For the case $\leq$ these inequalities can be stated as follows. 
Proposition 1 Let $S$ be a cover such that $\sum_{j \in S} c_{j}=d+\lambda, \lambda>0$ and $\bar{c}=$ $\max _{j \in S} c_{j}>\lambda$. Then the simple flow cover inequality

$$
\sum_{j \in S} x_{j}-\sum_{j \in S}\left(c_{j}-\lambda\right)^{+} z_{j} \leq d-\sum_{j \in S}\left(c_{j}-\lambda\right)^{+},
$$

defines a facet of $P^{1}$, and for $L \subseteq N \backslash S$ with $0<\bar{c}-\lambda<c_{k} \leq \bar{c}$ for all $k \in L$, the extended flow cover

$$
\sum_{j \in S \cup L} x_{j}-\sum_{j \in S}\left(c_{j}-\lambda\right)^{+} z_{j} \leq d-\sum_{j \in S}\left(c_{j}-\lambda\right)^{+}+\sum_{j \in L}(\bar{c}-\lambda) z_{j},
$$

defines a facet of $P^{1}$.

They showed that inequalities (2) together with the defining inequalities are enough to describe $P^{1}$ when $c_{j}=c, \forall j \in N$. Gu et al. [5] provided a strategy for sequence independent lifting of the flow cover inequalities using valid superadditive lifting functions. In particular, the lifted inequalities generalize inequalities (2).

Our main contribution is to extend the well-known polyhedral results for the SNFCN set to set $X$ and establish relations between the results for both. The paper is organized as follows. In Section 2 we establish basic properties of $P$, introduce a simple family of facet-defining inequalities and relate set $X$ with set $X^{1}$. In Section 3 we introduce the set-up flow cover inequalities and relate this class of inequalities with the well-known flow cover inequalities. We show that the new class of inequalities together with the inequalities defining $X$, and the simple family introduced in Section 2, give the complete characterization of $P$ when the capacities are constant. In Section 4 we discuss the lifting of the set-up flow cover inequalities. Preliminary computational experiments are reported in Section 5. Finally, main conclusions and future research are addressed in Section 6.

\section{Properties of $P$}

In this section we establish basic properties for $P$ and relate polyhedron $P$ with the SNFCN polyhedron.

We assume that for each $k \in N, 0<c_{k}<d$ and $\sum_{i=1}^{n} c_{i}>d+c_{k}$. Under these assumptions we trivially have the following result.

Proposition $2 P$ and $P^{1}$ are full dimensional polyhedra.

It can be easily checked that the inequalities $x_{j} \geq 0, z_{j} \leq 1, x_{j} \leq c_{j} z_{j}$ for all $j \in N, y \leq 1$ and $\sum_{j \in N} x_{j} \leq d y$ define facets of $P$. These are called trivial (facet-defining) inequalities.

Proposition 3 Consider a non-trivial facet-defining inequality

$$
\sum_{j \in N} \alpha_{j} x_{j}+\sum_{j \in N} \beta_{j} z_{j} \leq \delta y+\gamma
$$


Then (i) $\beta_{j} \leq 0, \forall j \in N$, (ii) $\gamma=0$, (iii) $\delta \geq 0$, (iv) $\alpha_{j} \geq 0, \forall j \in N$, (v) if $\beta_{j}<0$ then $\alpha_{j}>0, \forall j \in N$.

Proof Let $\mathcal{F}$ be the facet defined by (3). Proof of (i). Suppose $\beta_{j}>0$. If there exists a point $\left(x^{*}, z^{*}, y^{*}\right) \in \mathcal{F} \cap X$ satisfying $z_{j}^{*}=0$, then the point $\left(x^{*}, z^{\prime}, y^{*}\right) \in$ $X$ where $z_{j}^{\prime}=1, z_{k}^{\prime}=z_{k}^{*}, k \neq j$ violates (3). Hence, $\mathcal{F} \subseteq\left\{(x, z, y) \mid z_{j}=1\right\}$, which is a contradiction.

Proof of (ii). Since $(\mathbf{0}, \mathbf{0}, 0) \in X$ and inequality (3) is valid for $X$, then $\gamma \geq 0$. Suppose $\gamma>0$. Since there can be no point in $\mathcal{F}$ with $y=0$ (because $x_{j}=0, j \in N$ and $\left.\beta_{j} \leq 0, j \in N\right)$ then $y=1, \forall(x, z, y) \in \mathcal{F}$. Thus, $\mathcal{F} \subseteq$ $\{(x, z, y) \mid y=1\}$, which is a contradiction.

Proof of (iii). Since $(\mathbf{0}, \mathbf{0}, 1) \in X, \gamma=0$, and (3) is valid for $X$, then $\delta \geq 0$.

Proof of (iv). Suppose to the contrary that $\alpha_{j}<0$ for some $j \in N$. There must exist a point $\left(x^{*}, z^{*}, y^{*}\right) \in \mathcal{F} \cap X$ satisfying $x_{j}^{*}>0$, since otherwise $\mathcal{F} \subseteq$ $\left\{(x, z, y) \mid x_{j}=0\right\}$. As $\left(x^{*}, z^{*}, y^{*}\right) \in \mathcal{F}$, then $\sum_{i \in N} \alpha_{i} x_{i}^{*}+\sum_{i \in N} \beta_{i} z_{i}^{*}=\delta y^{*}$. Then we generate a new point $\left(x^{\prime}, z^{*}, y^{*}\right) \in X$ such that $x_{i}^{\prime}=x_{i}^{*}, \forall i \neq j, x_{i}^{\prime}=$ 0 . Clearly, $\left(x^{\prime}, z^{*}, y^{*}\right) \in X$ violates inequality (3). Therefore $\alpha_{j} \geq 0, \forall j \in N$.

Proof of (v). Suppose that for some $j \in N, \beta_{j}<0$ and $\alpha_{j}=0$. Then as in the proof of (iv) we can show that all the points in $\mathcal{F}$ satisfy $z_{j}=0$ and so $\mathcal{F} \subseteq\left\{(x, z, y) \mid z_{j}=0\right\}$, which is a contradiction.

Set $X$ is very closely related to $X^{1}$. The following property relates valid inequalities for the two sets: $X$ and $X^{1}$.

Proposition 4 Consider the following inequality

$$
\sum_{j \in N} \alpha_{j} x_{j}+\sum_{j \in N} \beta_{j} z_{j} \leq \delta,
$$

(i) If (4) is valid for $X^{1}$, then (4) is valid for $X$.

(ii) If $\beta_{j} \leq 0, \forall j \in N$, then inequality (4) is valid for $X^{1}$, if and only if

$$
\sum_{j \in N} \alpha_{j} x_{j}+\sum_{j \in N} \beta_{j} z_{j} \leq \delta y,
$$

is valid for $X$.

Proof (i) Suppose $(x, z, y) \in X$ violates (4). Hence $y=0$, which implies $x_{j}=$ $0, \forall j \in N$. Thus, the point $(\mathbf{0}, z, 1) \in X^{1}$ and violates inequality (4), which is a contradiction.

(ii) Consider $(x, z, y) \in X$ and suppose that (4) is valid for $X^{1}$. If $y=1$, then validity of (4) implies that $(x, z, y)$ satisfies (5). If $y=0$, then $\sum_{j \in N} x_{j} \leq$ $d y=0$ and $x_{j} \geq 0$ imply that $x_{j}=0, \forall j \in N$. Since $\beta_{j} \leq 0$ and $z_{j} \geq 0$, then $\sum_{j \in N} \beta_{j} z_{j} \leq 0$ which shows that $(x, z, y)$ satisfies (5). Now suppose that (5) is valid for $X$. Since $X^{1}$ is a restriction of $X$ with $y=1$ so (4) is valid for $X^{1}$. 
From Proposition 4, part (i), it follows that the flow covers are valid for $X$. Moreover, the inequalities

$$
\sum_{j \in S} x_{j}-\sum_{j \in S^{+}}\left(c_{j}-\lambda\right) z_{j} \leq\left(d-\sum_{j \in S^{+}}\left(c_{j}-\lambda\right)\right) y, \forall S \subseteq N
$$

where $S$ is a cover and $S^{+}=\left\{j \in S: c_{j}>\lambda\right\}$, are valid for $X$. Inequalities (6) can be regarded as strengthened simple flow cover inequalities.

Observing that the point $(x, z, y)=(\mathbf{0}, \mathbf{0}, 0)$ satisfies $(5)$ as equation, it is straightforward to check the following result.

Proposition 5 If (4) defines a facet of $P^{1}$, then (5) defines a facet of $P$.

However, the structure of $P$ is richer than the structure of $P^{1}$ since it includes many new facet-defining inequalities. Next we introduce a new family of facet-defining inequalities.

Proposition 6 The inequality

$$
x_{j} \leq c_{j} y, j \in N \text {, }
$$

is valid for $X$ and defines a non-trivial facet of $P$.

Proof The proof of validity is trivial. To show it defines a facet consider the following $2 n+1$ affinely independent points: $(\mathbf{0}, \mathbf{0}, 0),\left(\mathbf{0}, e_{k}, 0\right), k \in N$, $\left(c_{j} e_{j}, e_{j}, 1\right),\left(c_{j} e_{j}+b_{k} e_{k}, e_{j}+e_{k}, 1\right), k \in N \backslash\{j\}$, where $b_{k}=\min \left\{d-c_{j}, c_{k}\right\}$ and $e_{k}$ is the vector with 1 in component $k$ and 0 elsewhere.

\section{Set-up flow cover inequalities}

In this section we introduce the set-up flow cover inequalities which can be seen an extension of the flow cover inequalities to set $X$.

Proposition 7 Let $S$ be a cover with $\max _{j \in S} c_{j}>\lambda$. For each $\emptyset \neq \bar{S}^{+} \subseteq$ $S^{+}=\left\{j \in S: c_{j}>\lambda\right\}$, the simple set-up flow cover inequality

$$
\sum_{j \in S} x_{j}-\sum_{j \in \bar{S}^{+}}\left(c_{j}-\lambda\right) z_{j} \leq\left(d-\sum_{j \in \bar{S}^{+}}\left(c_{j}-\lambda\right)\right) y,
$$

is a facet of $P$.

Proof First, we justify the validity. If $y=0$, then $x_{j}=0, \forall j \in N$. Since, for $j \in \bar{S}^{+}, c_{j}-\lambda>0$ and $z_{j} \geq 0$, then $-\sum_{j \in \bar{S}^{+}}\left(c_{j}-\lambda\right) z_{j} \leq 0$, which implies (8). Now assume $y=1$. Let $(x, z, y)$ be a point of $X$ with $z_{i}=1$ for $i \in T$, and $z_{i}=0$ otherwise. We consider the following cases.

Case 1. $\left|\bar{S}^{+} \backslash T\right|=0$. It implies $z_{j}=1, \forall j \in \bar{S}^{+}$and so the validity of (8) follows from $\sum_{j \in S} x_{j} \leq d$ clearly. 
Case 2. $\left|\bar{S}^{+} \backslash T\right| \geq 1$. Then $\sum_{j \in S} x_{j}-\sum_{j \in \bar{S}^{+}}\left(c_{j}-\lambda\right) z_{j}=\sum_{j \in S \cap T} x_{j}-$ $\sum_{j \in \bar{S}^{+} \cap T}\left(c_{j}-\lambda\right) \leq \sum_{j \in S \cap T} c_{j}-\sum_{j \in \bar{S}^{+} \cap T} c_{j}+\left|\bar{S}^{+} \cap T\right| \lambda=\sum_{j \in S \cap T} c_{j}+$ $\sum_{j \in \bar{S}^{+} \backslash T} c_{j}-\sum_{j \in \bar{S}^{+}} c_{j}-\lambda+\left(\left|\bar{S}^{+} \cap T\right|+1\right) \lambda \leq \sum_{j \in S} c_{j}-\lambda-\sum_{j \in \bar{S}^{+}} c_{j}+$ $\left(\left|\bar{S}^{+} \cap T\right|+1\right) \lambda=d-\sum_{j \in \bar{S}^{+}} c_{j}+\left(\left|\bar{S}^{+} \cap T\right|+1\right) \lambda \leq d-\sum_{j \in \bar{S}^{+}}\left(c_{j}-\lambda\right)$, where the last inequality follows from $\left|\bar{S}^{+} \backslash T\right| \geq 1$ which implies $\left|\bar{S}^{+} \cap T\right| \leq\left|\bar{S}^{+}\right|-1$.

To prove (8) defines a facet we construct $2 n+1$ affinely independent points of the form $\left(X_{S \backslash \bar{S}^{+}}, X_{\bar{S}^{+}}, X_{N \backslash S}, Z_{S \backslash \bar{S}^{+}}, Z_{\bar{S}^{+}}, Z_{N \backslash S}, y\right)$, satisfying (8) as equation, where $X_{J}$ is the vector of $x_{j}$ 's for $j \in J \subseteq N$. Since $S$ is a cover, there exist $s=|S|$ affinely independent points $\left(X_{S \backslash \bar{S}^{+}}^{k}, X_{\bar{S}^{+}}^{k}\right), k \in S$ satisfying $0 \leq x_{j} \leq$ $c_{j}$ for $j \in S$ and $\sum_{j \in S} x_{j}=d$. We assume $S=\{1, \ldots s\}$. Now, for $k \in \bar{S}^{+}$, let $l_{k}=\max \left\{\sum_{j \in S} x_{j}-\sum_{j \in \bar{S}^{+}}\left(c_{j}-\lambda\right) z_{j}+\sum_{j \in \bar{S}^{+} \backslash\{k\}}\left(c_{j}-\lambda\right) \mid \sum_{j \in S} x_{j} \leq d, x_{j} \leq\right.$ $\left.c_{j} z_{j}, j \in S, z_{j} \in\{0,1\}, j \in S, z_{k}=0\right\}$ and let $\bar{X}^{k}=\left(\bar{X}_{S \backslash \bar{S}^{+}}^{k}, \bar{X}_{\bar{S}^{+}}^{k}\right)$ be an optimal solution of this maximization problem. From validity of (8), and $z_{k}=0$, we have $l_{k}+\left(c_{k}-\lambda\right) \leq d$. On the other hand, as $c_{k}>\lambda$, then $\sum_{j \in S \backslash\{k\}} c_{j} \leq d$. Hence, considering the solution $z_{j}=1$, and $x_{j}=c_{j}$ for all $j \in S \backslash\{k\}, y=1$, and $z_{k}=x_{k}=0$, we have $l_{k} \geq d-\left(c_{k}-\lambda\right)$. Thus, $l_{k}=d-\left(c_{k}-\lambda\right)$.

Combining the assumptions $\sum_{j \in N} c_{j}>d+c_{k}$ and $\max _{j \in S} c_{j}>\lambda$ gives $S \varsubsetneqq N$. Without loss of generality, assume that $1 \in \bar{S}^{+}$. For each vector $\bar{X}^{k}$ with the property $\sum_{j \in S} \bar{X}_{j}^{k}=d-\left(c_{k}-\lambda\right)$, we define $\varepsilon_{k}>0$ such that $\sum_{j \in S} \bar{X}_{j}^{k}+\varepsilon_{k} \sum_{j \in N \backslash S} c_{j}=d$. In fact, $\varepsilon_{k}=\left(c_{k}-\lambda\right) /\left(\sum_{j \in N \backslash S} c_{j}\right)$.

Let $e_{j}$ denote the $j$ th unit vector, 1 denote the vector whose components are all one, and $\mathbf{0}$ denote the vector whose components are all zero. Then consider the following points:
(i) $\left(X_{S \backslash \bar{S}^{+}}^{k}, X_{\bar{S}^{+}}^{k}, \mathbf{0}, \mathbf{1}, \mathbf{1}, \mathbf{0}, 1\right)$,
$k \in S$,
(ii) $\left(\bar{X}_{S \backslash \bar{S}^{+}}^{k}, \bar{X}_{\bar{S}^{+}}^{k}, \mathbf{0}, \mathbf{1}, \mathbf{1}-e_{k}, \mathbf{0}, 1\right)$,
$k \in \bar{S}^{+}$,
(iii) $\left(\mathbf{0}, \mathbf{0}, \mathbf{0}, \mathbf{0}, \mathbf{0}, e_{j}, 0\right)$
$j \in N \backslash S$,
(iv) $\left(\bar{X}_{S \backslash S^{+}}^{1}, \bar{X}_{\bar{S}^{+}}^{1}, \varepsilon_{1} c_{j} e_{j}, \mathbf{1}, \mathbf{1}-e_{1}, e_{j}, 1\right), \quad j \in N \backslash S$,
(v) $\left(\mathbf{0}, \mathbf{0}, \mathbf{0}, e_{k}, \mathbf{0}, \mathbf{0}, 0\right)$,
$k \in S \backslash \bar{S}^{+}$,
(vi) $(\mathbf{0}, \mathbf{0}, \mathbf{0}, \mathbf{0}, \mathbf{0}, \mathbf{0}, 0)$.

The set of given points belong to $X$ and satisfies inequality (8) at equality. Suppose that these points lie on the following hyperplane.

$$
\sum_{j \in N} \alpha_{j} x_{j}+\sum_{j \in N} \beta_{j} z_{j}=\gamma y+\gamma_{0} .
$$

Substituting point (vi) in hyperplane (9) gives $\gamma_{0}=0$. Using points of type (iii) and (v) we have $\beta_{j}=0, j \in N \backslash \bar{S}^{+}$. Since points (i) lie in the hyperplane $\sum_{j \in S} \alpha_{j} x_{j}+\sum_{j \in \bar{S}^{+}} \beta_{j}=\gamma$, and uniquely define $\sum_{j \in S} x_{j}=d$, then $\alpha_{j}=$ $\alpha, j \in S$ and $\alpha d+\sum_{j \in \bar{S}^{+}} \beta_{j}=\gamma$. Considering the point of type (ii) with $k=1$ and points in (iv) we obtain $\alpha_{j}=0, j \in N \backslash S$. By substituting the points (ii) in (9) it follows that $\alpha \sum_{j \in S} \bar{X}_{j}^{k}+\sum_{j \in \bar{S}^{+}} \beta_{j}-\beta_{k}=\gamma$, for $k \in \bar{S}^{+}$. Combining 
this equation with $\alpha d+\sum_{j \in \bar{S}^{+}} \beta_{j}=\gamma$ and $\sum_{j \in S} \bar{X}_{j}^{k}=d-\left(c_{k}-\lambda\right)$ implies $\beta_{k}=-\alpha\left(c_{k}-\lambda\right), k \in \bar{S}^{+}$. Finally, using any point of type (i) it follows that $\gamma=\alpha\left(d-\sum_{j \in \bar{S}^{+}}\left(c_{j}-\lambda\right)\right)$. Hence, $(9)$ is a positive multiple of the hyperplane defined by (8).

Notice that the simple flow covers can be obtained from (8), setting $y=1$ and considering $\bar{S}^{+}=S^{+}$.

Next we give the extended set-up flow cover inequalities. The result is given without proof since it can be derived from the lifting of inequalities (8) discussed in Section 4.

Proposition 8 Let $S$ be a cover with $\max _{j \in S} c_{j}>\lambda$. For each $\emptyset \neq \bar{S}^{+} \subseteq$ $S^{+}=\left\{j \in S: c_{j}>\lambda\right\}$, and for each $L \subseteq N \backslash S$ where for $k \in L, \bar{c}-\lambda<c_{k} \leq \bar{c}$, and $\bar{c}=\max _{j \in \bar{S}^{+}} c_{j}$, the extended set-up flow cover inequality

$$
\sum_{j \in S \cup L} x_{j}-\sum_{j \in \bar{S}^{+}}\left(c_{j}-\lambda\right) z_{j}-\sum_{j \in L}(\bar{c}-\lambda) z_{j} \leq\left(d-\sum_{j \in \bar{S}^{+}}\left(c_{j}-\lambda\right)\right) y,
$$

is a facet of $P$.

When the capacities are constant $\left(c_{j}=c, \forall j \in N\right)$ we obtain the following class of inequalities.

Corollary 1 Assume $c_{j}=c, \forall j \in N, d>c>0, n c>d$, and assume $d$ is not a multiple of c. Define $r=d-\left\lfloor\frac{d}{c}\right\rfloor c$. Let $S_{1}, S_{2} \subseteq N$ such that $S_{1} \cap S_{2}=\emptyset$ and $\left|S_{1}\right|<\left\lceil\frac{d}{c}\right\rceil,\left\lceil\frac{d}{c}\right\rceil \leq\left|S_{1}\right|+\left|S_{2}\right|$. Then the following inequality is non-trivial facet of $P$.

$$
\sum_{j \in S_{1}} x_{j}+\sum_{j \in S_{2}}\left(x_{j}-r z_{j}\right) \leq(d-k r) y
$$

where $k=\left\lceil\frac{d}{c}\right\rceil-\left|S_{1}\right|$.

Example 1 Consider an instance with $n=4, d=14$, and $c=5$. So $r=4$. Using the software PORTA [3], we obtain 18 facet-defining inequalities for $P^{1}$ and 57 facet-defining inequalities for $P$. The non-trivial facet-defining inequalities for $P^{1}$ are the following.

$$
\begin{aligned}
& x_{2}+x_{3}+x_{4}-4 z_{2}-4 z_{3}-4 z_{4} \leq 2, \\
& x_{1}+x_{3}+x_{4}-4 z_{1}-4 z_{3}-4 z_{4} \leq 2, \\
& x_{1}+x_{2}+x_{4}-4 z_{1}-4 z_{2}-4 z_{4} \leq 2, \\
& x_{1}+x_{2}+x_{3}-4 z_{1}-4 z_{2}-4 z_{3} \leq 2, \\
& x_{1}+x_{2}+x_{3}+x_{4}-4 z_{1}-4 z_{2}-4 z_{3}-4 z_{4} \leq 2 .
\end{aligned}
$$

For $P$ we have 43 non-trivial inequalities. For instance, considering $S=$ $\{1,2,3\}$, we have the following facet-defining inequalities of type (11) for $k=$ 1,2 , and 3 :

$$
\begin{aligned}
& x_{1}+x_{2}+x_{3}-4 z_{2} \leq 10 y, \quad k=1, \\
& x_{1}+x_{2}+x_{3}-4 z_{2}-4 z_{3} \leq 6 y, \quad k=2, \\
& x_{1}+x_{2}+x_{3}-4 z_{1}-4 z_{2}-4 z_{3} \leq 2 y, \quad k=3 .
\end{aligned}
$$


Note that for $k=3$, the inequality appears in $P^{1}$ as a facet-defining inequalities by setting $y=1$. However for $k=1$ and $k=2$ the corresponding inequalities for $P^{1}$, obtained by setting $y=1$, are not facet-defining.

Next we give the full polyhedral description of $P$ when the $c_{j}=c, \forall j \in N$.

Theorem 1 If $c_{j}=c, j \in N$, the defining inequalities of $X$ with inequalities (7) and (11) suffice to describe $P$.

Proof Set $X$ can be decomposed into two mixed-integer sets whose polyhedral characterization is known: set $X^{1}$, obtained by restricting $y=1$, and set $X^{0}$ obtained by restricting $y=0$. The convex hull of $X^{1}$, denoted by $P^{1}$, was derived in [7], and is given by the trivial facet-defining inequalities and the simple flow cover inequalities. The convex hull of $X^{0}, P^{0}$, is given by $P^{0}=\left\{(x, z, y) \in \mathbb{R}^{2 n+1}: x_{j}=0, j \in N, 0 \leq z_{j} \leq 1, j \in N, y=0\right\}$. Polyhedron $P$ is the closed convex hull of $P^{0} \cup P^{1}$ and can be represented as a linear program in a higher dimensional space (see [2]) as follows:

$$
\begin{aligned}
& \left\{\left(x, z, y, x^{0}, z^{0}, y^{0}, x^{1}, z^{1}, y^{1}, \delta_{0}, \delta_{1}\right) \in \mathbb{R}^{6 n+5}:\right. \\
& x_{j}^{0}=0, j \in N, 0 \leq z_{j}^{0} \leq \delta^{0}, j \in N, y^{0}=0, \\
& x_{j}^{1} \geq 0, j \in N, x_{j}^{1} \leq c z_{j}^{1}, j \in N, z_{j}^{1} \leq \delta^{1}, j \in N, \sum_{j \in N} x_{j}^{1} \leq d \delta^{1}, y^{1}=\delta^{1}, \\
& \sum_{j \in S}\left(x_{j}^{1}-r z_{j}^{1}\right) \leq\left(d-r\left\lceil\frac{d}{c}\right\rceil\right) \delta^{1}, \forall S \subseteq N:|S| \geq\left\lceil\frac{d}{c}\right\rceil, \\
& \left.x_{j}=x_{j}^{0}+x_{j}^{1}, z_{j}=z_{j}^{0}+z_{j}^{1}, y=y^{0}+y^{1}, \delta^{0}+\delta^{1}=1\right\} .
\end{aligned}
$$

Projecting out variables $x_{j}^{0}, x_{j}^{1}, z_{j}^{1}, j \in N, \delta^{0}, \delta^{1}, y^{0}, y^{1}$ (using the equations $\left.x_{j}^{0}=0, x_{j}^{1}=x_{j}-x_{j}^{0}, z_{j}^{1}=z_{j}-z_{j}^{0}, \delta^{0}=1-\delta^{1}, \delta^{1}=y^{1}, y^{0}=0, y^{1}=y-y^{0}\right)$ we obtain: $\left\{\left(x, z, y, z^{0}\right) \in \mathbb{R}^{3 n+1}\right.$ :

$$
\begin{aligned}
& x_{j} \geq 0, j \in N, \\
& \sum_{j \in N} x_{j} \leq d y, \\
& \sum_{j \in S}\left(x_{j}-r z_{j}+r z_{j}^{0}\right) \leq\left(d-r\left\lceil\frac{d}{c}\right\rceil\right) y, \forall S \subseteq N:|S| \geq\left\lceil\frac{d}{c}\right\rceil, \\
& z_{j}-z_{j}^{0} \leq y, j \in N, \\
& z_{j}^{0} \leq 1-y, j \in N, \\
& x_{j} \leq c\left(z_{j}-z_{j}^{0}\right), j \in N, \\
& \left.z_{j}^{0} \geq 0, j \in N\right\} .
\end{aligned}
$$

Now we use the Fourier-Motzkin elimination (see [6]) to project out variables $z_{j}^{0}, j \in N$. Inequalities (15) and (16) imply $z_{j} \leq 1, \forall j \in N$; inequalities (15) and (17) imply $x_{j} \leq c y \forall j \in N$; (16) and (18) imply $y \leq 1$; (17) and (18) imply $x_{j} \leq c z_{j}, \forall j \in N$. Finally, combining (14), with (15) for $j \in S_{1} \subseteq S$ 
and (18) for $j \in S_{2}=S \backslash S_{1}$ we have (11). Notice that when $\left|S_{1}\right| \geq\left\lfloor\frac{d}{c}\right\rfloor$ the projected inequality does not define a facet. Hence, the projected polyhedron is $P$.

Next we explain the relation between the polyhedra defined by the simple flow covers $(1), P_{S F C}$, the strengthened simple flow covers $(6), P_{S F C}^{Y}$, and the polyhedron defined by the simple set-up flow covers (8), $P_{S S F C}^{Y}$.

Proposition 9 The inclusions $P_{S S F C}^{Y} \subseteq P_{S F C}^{Y} \subseteq P_{S F C}$ hold. Moreover, $P_{S F C}^{Y} \bigcap\left\{(x, z, y): z_{j} \leq y, \forall j \in N\right\}=P_{S S F C}^{Y} \cap\left\{(x, z, y): z_{j} \leq y, \forall j \in N\right\}$.

Proof The first two inclusions are trivial. Suppose $z_{j} \leq y, \forall j \in N$. Since $P_{S S F C}^{Y} \subseteq P_{S F C}^{Y}$ we have $P_{S F C}^{Y} \bigcap\left\{(x, z, y): z_{j} \leq y, \forall j \in N\right\} \supseteq P_{S S F C}^{Y} \cap$ $\left\{(x, z, y): z_{j} \leq y, \forall j \in N\right\}$. To prove the inclusion $\subseteq$ we show that inequalities (8) with $\bar{S}^{+} \subsetneq S^{+}$do not define facets. For each $\emptyset \subsetneq \bar{S}^{+} \subsetneq S^{+}$we have $\sum_{j \in S^{+} \backslash \bar{S}^{+}} z_{j} \leq \sum_{j \in S^{+} \backslash \bar{S}^{+}} y$. Thus, $\sum_{j \in S^{+} \backslash \bar{S}^{+}}\left(c_{j}-\lambda\right) z_{j} \leq \sum_{j \in S^{+} \backslash \bar{S}^{+}}\left(c_{j}-\lambda\right) y$. Adding this inequality to (6) (which is (8) with $\bar{S}^{+}=S^{+}$) we obtain the set-up flow cover (8) defined by $S^{+}$and $\bar{S}^{+}$.

Restrictions $z_{j} \leq y$ occur in some practical problems where a set-up of an arc can occur only if the node is open, see for example [1]. Proposition 9 states that in such cases inequalities with $\bar{S}^{+} \subsetneq S^{+}$are dominated and it suffices to strengthen the flow covers $(6)$ to get the non-dominated inequalities.

Example 2 Consider the data in Example 1 and the fractional solution $y^{*}=$ $0.7, z_{1}^{*}=1, z_{2}^{*}=1, z_{3}^{*}=0.5, z_{4}^{*}=0, x_{1}^{*}=5, x_{2}^{*}=2.3, x_{3}^{*}=2.5, x_{4}^{*}=0$. There is no flow cover inequality (2) and no strengthened flow cover inequality (6) cutting off the extreme point. However the inequality $x_{1}+x_{2}+x_{3}-4 z_{3} \leq 10 y$ is violated.

Now we consider the separation problem associated with the set-up flow cover inequalities. Consider a fractional solution $\left(x^{*}, z^{*}, y^{*}\right)$. If there is an inequality (8) cutting off $\left(x^{*}, z^{*}, y^{*}\right)$ for a given set $S$ and $S^{+}$, the most violated inequality is obtained by considering $\bar{S}^{+}=\left\{j \in S^{+} \mid z_{j}^{*} \leq y^{*}\right\}$. Thus, any separation heuristic for flow covers directly leads to a separation heuristic for inequalities (8). Following [7], for each $\lambda$ one can find the most violated inequality (8) by solving the knapsack problem:

$$
\eta_{\lambda}=\max \quad\left\{\sum_{j \in N} \tau_{j}(\lambda) w_{j} \mid \sum_{j \in N} c_{j} w_{j}=d+\lambda, \quad w_{j} \in\{0,1\}, j \in N\right\},
$$

where $\tau_{j}(\lambda)=x_{j}^{*}+\left(c_{j}-\lambda\right)^{+} \times\left(y^{*}-z_{j}^{*}\right)^{+}$. Let $U=\left\{j \in N \mid w_{j}=1\right\}$ and $\bar{U}^{+}=\left\{j \in U \mid c_{j}>\lambda \wedge y^{*}>z_{j}^{*}\right\}$. If $\eta_{\lambda}>d y^{*}$ then a violated inequality (8) with $S=U$ and $\bar{S}^{+}=\bar{U}^{+}$has been found. Otherwise, no such violated inequality exists.

In the constant capacitated case, the separation of (11) amounts to checking whether $\max _{S_{1}, S_{2} \subseteq N, S_{1} \cap S_{2}=\emptyset, k=\left\lceil\frac{d}{c}\right\rceil-\left|S_{1}\right|}\left\{\sum_{j \in S_{1}} x_{j}^{*}+\sum_{j \in S_{2}}\left(x_{j}^{*}-r z_{j}^{*}\right)+\right.$ 
$\left.k r y^{*}\right\}$ is strictly greater than $d y^{*}$ (the inequality induced by $S_{1}, S_{2}$, and $k$ is violated) or not. In the last case there are no violated inequalities in this family.

The foregoing maximization problem is equivalent to the following integer program.

$$
\begin{aligned}
(I P) \max \left\{\sum_{j \in N}\left(u_{j} x_{j}^{*}+v_{j}\left(x_{j}^{*}-r z_{j}^{*}\right)\right)+k r y^{*} \mid \sum_{j \in N} u_{j}+k=\left\lceil\frac{d}{c}\right\rceil,\right. \\
\left.u_{j}+v_{j} \leq 1, j \in N, k \leq\left\lceil\frac{d}{c}\right\rceil, u_{j}, v_{j} \in\{0,1\}, j \in N, k \in \mathbb{Z}_{0}^{+}\right\},
\end{aligned}
$$

where $u_{j}=1$ if and only if $j \in S_{1}$ and $v_{j}=1$ if and only if $j \in S_{2}$. This integer program (IP) is a special case of the Transportation Problem for which there are very efficient combinatorial algorithms.

\section{Lifting the set-up flow cover inequalities}

In this section we discuss the lifting of inequalities (8), following the approach presented in [5].

For $T \subset N$, let $\bar{X}=\left\{(x, z, y) \in X \mid\left(x_{j}, z_{j}\right)=(0,0), j \in T\right\}$ and consider a valid inequality (8) for $\bar{X}$. For a given variable pair $\left(x_{k}, z_{k}\right), k \in T$, we want to determine the coefficients $\alpha_{k}, \beta_{k}$ such that the inequality

$$
\sum_{j \in S} x_{j}+\alpha_{k} x_{k}-\sum_{j \in \bar{S}^{+}}\left(c_{j}-\lambda\right) z_{j}+\beta_{k} z_{k} \leq\left(d-\sum_{j \in \bar{S}^{+}}\left(c_{j}-\lambda\right)\right) y,
$$

is valid for $\bar{X}^{k}=\left\{(x, z, y) \in X \mid\left(x_{j}, z_{j}\right)=(0,0), j \in T \backslash\{k\}\right\}$. Let

$$
h_{k}(u)=\max \left\{\alpha_{k} x_{k}+\beta_{k} z_{k} \mid x_{k}=u, 0 \leq x_{k} \leq c_{k} z_{k}, z_{k} \in\{0,1\}\right\},
$$

and consider the lifting function:

$$
\begin{aligned}
f(u)=\min & \left(d-\sum_{j \in \bar{S}^{+}}\left(c_{j}-\lambda\right)\right) y-\sum_{j \in S} x_{j}+\sum_{j \in \bar{S}^{+}}\left(c_{j}-\lambda\right) z_{j} \\
\text { s.t. } & \sum_{j \in N \backslash T} x_{j} \leq d y-u, \\
& 0 \leq x_{j} \leq c_{j} z_{j}, j \in N \backslash T, \\
& z_{j} \in\{0,1\}, j \in N \backslash T, \\
& y \in\{0,1\},
\end{aligned}
$$

where $S \subseteq N \backslash T$ is a cover with $\max _{j \in S} c_{j}>\lambda$. Then inequality (19) is valid for $\bar{X}^{k}$ if and only if $h_{k}(u) \leq f(u), 0 \leq u \leq c_{k}$. Moreover, in order to obtain a strongest lifted inequality (known as maximal lifting), $\alpha_{k}$ and $\beta_{k}$ should be such that the equation $h_{k}(u)=f(u)$ has two linearly independent solutions. If 
(8) defines a facet for $\operatorname{conv}(\bar{X})$ and the lifting is maximal, then the resulting inequality defines a facet for $\operatorname{conv}\left(\bar{X}^{k}\right)$.

First we characterize function $f$. Feasibility of the lifting problem associated with the lifting function $f(u)$, for $u>0$, implies $y=1$ because $x_{j} \geq 0, j \in N \backslash T$ and $\sum_{j \in N \backslash T} x_{j} \leq d y-u$. Hence the lifting function is similar to the one given in $[5]$, p. 450 , for the flow covers on $[0, d]$.

Let $\bar{S}^{+}=\left\{\ell_{1}, \ldots, \ell_{r}\right\}$ with $c_{\ell_{i}} \geq c_{\ell_{i+1}}$ for $i=1, \ldots, r-1$. Function $f$, for $u \geq 0$, can be written as

$$
f(u)= \begin{cases}i \lambda, & M_{i} \leq u \leq M_{i+1}-\lambda, i=0, \ldots, r-1, \\ u-M_{i}+i \lambda, & M_{i}-\lambda \leq u \leq M_{i}, i=1, \ldots, r-1, \\ u-M_{r}+r \lambda, & M_{r}-\lambda \leq u \leq d,\end{cases}
$$

where $M_{0}=0$ and $M_{i}=\sum_{k=1}^{i} c_{\ell_{k}}$ for $i=1, \ldots, r$.

From Theorem 6 in [5], function $f$ is superadditive on $[0, d]$. Hence, the lifting of all variable pairs $\left(x_{j}, z_{j}\right), j \in T$ can be done simultaneously. Different functions $h_{j}(u)$ can be defined for each $j \in T$, leading to maximal lifted inequalities. For each $j \in T$ we define $h_{j}(u)$ as a line passing through the points $\left(u, h_{j}(u)\right)$ for $u=c_{j}$ and $u=M_{i}-\lambda$ where $i=\operatorname{argmax}\left\{t \in\{1, \ldots, r\} \mid M_{t}-\lambda \leq\right.$ $\left.c_{j}\right\}$. It can be easily checked that $h_{j}(u)$ underestimates $f$ in $\left[0, c_{j}\right]$. From this discussion, and computing the values of $\alpha_{j}, \beta_{j}$ such that $h_{j}(u)=f(u)$ for the two points given above, it follows that the following inequalities are valid for $X$.

$$
\sum_{j \in S} x_{j}+\sum_{j \in T} \alpha_{j} x_{j}-\sum_{j \in \bar{S}^{+}}\left(c_{j}-\lambda\right) z_{j}+\sum_{j \in T} \beta_{j} z_{j} \leq\left(d-\sum_{j \in \bar{S}^{+}}\left(c_{j}-\lambda\right)\right) y,
$$

where, $\left(\alpha_{j}, \beta_{j}\right)=\left(\frac{\lambda}{c_{j}-M_{i}+\lambda},(i-1) \lambda-\frac{\lambda\left(M_{i}-\lambda\right)}{c_{j}-M_{i}+\lambda}\right)$ if $M_{i} \leq c_{j} \leq M_{i+1}-\lambda$, and $\left(\alpha_{j}, \beta_{j}\right)=\left(1, i \lambda-M_{i}\right)$ otherwise. Inequalities (20) imply (10) since, for $j \in L$, $\bar{c}-\lambda \leq c_{j} \leq \bar{c}$, where $\bar{c}=\max \left\{c_{j} \mid j \in \bar{S}^{+}\right\}$.

An interesting question arises when we consider more general sets $X^{\prime}$ obtained from $X$ replacing the inequality $\sum_{j \in N} x_{j} \leq d y$ by $\sum_{j \in N} x_{j}-\sum_{j \in N^{-}} x_{j} \leq$ $d y$ or by $\sum_{j \in N} x_{j} \leq d y+s$ with $s \geq 0$. In both cases (8) is valid for the restriction of $X^{\prime}$ to the subspace defined by $\left(x_{j}, z_{j}\right)=(0,0), j \in T \cup N^{-}$, or $s=0$, respectively. For these cases, in order to lift (8), we need to consider $f(u)$ for $u<0$. For negative $u$, the minimum of the lifting function is obtained by setting $y=0$. For example, as long as $u \geq-\sum_{j \in S \backslash \bar{S}^{+}} c_{j}$, the value of $f(u)$ is obtained by setting $y=0, x_{j}=z_{j}=0, j \in \bar{S}^{+}$and $\sum_{j \in S \backslash \bar{S}^{+}} x_{j}=u$. Hence, for $u<0$, we have

$f(u)= \begin{cases}-\gamma-r \lambda, & u \leq-\sum_{j \in S} c_{j}, \\ u+N_{j}-j \lambda, & -\gamma-N_{j} \leq u \leq-\gamma-N_{j}+\lambda, j=1, \ldots, r, \\ -\gamma-j \lambda, & -\gamma-N_{j+1}+\lambda \leq u \leq-\gamma-N_{j}, j=0, \ldots, r-1, \\ u, & -\gamma \leq u \leq 0,\end{cases}$

where $\gamma=\sum_{j \in S \backslash \bar{S}^{+}} c_{j}, N_{0}=0$ and $N_{j}=\sum_{k=r-j+1}^{r} c_{\ell_{k}}$ for $j=1, \ldots, r$. 
Function $f(u)$ is not superadditive in all its domain. In order to perform a simultaneous lifting we derive superadditive functions that underestimate $f$ (called valid lifting functions). Since $f(u)$ is superadditive for $u \geq 0$, one such function can be obtained by underestimating $f$ on the negative side: $g_{1}(u)= \begin{cases}u & u<0, \\ f(u) & u \geq 0 .\end{cases}$

Proposition 10 Function $g_{1}$ is a valid superadditive lifting function for $f$.

Proof Since $g_{1}$ is superadditive on $[0, d]$ and on $[-\infty, 0]$, separately, we only need to prove that $g\left(u_{1}\right)+g\left(u_{2}\right) \leq g\left(u_{1}+u_{2}\right)$ when $u_{1}$ and $u_{2}$ have opposite signs. Assume $u_{1}<0$ and $M_{i} \leq u_{2} \leq M_{i+1}-\lambda$.

If $u_{1}+u_{2}<0$, then $g_{1}\left(u_{1}+u_{2}\right)=u_{1}+u_{2} \geq u_{1}+M_{i}=u_{1}+\sum_{t=1}^{i} c_{\ell_{t}} \geq u_{1}+i \lambda$ $=g_{1}\left(u_{1}\right)+g_{1}\left(u_{2}\right)$.

Now assume $u_{1}+u_{2} \geq 0$. Case 1: $M_{j} \leq u_{1}+u_{2} \leq M_{j+1}-\lambda$, for some $j \leq i$. As $u_{1}+u_{2} \leq M_{j+1}-\lambda$ and $-u_{2} \leq-M_{i}$, then $u_{1} \leq M_{j+1}-\lambda-M_{i}$ $=M_{j+1}-\lambda-M_{j+1}-\sum_{t=j+2}^{i} c_{\ell_{t}} \leq-\lambda-(i-j-1) \lambda=(j-i) \lambda$. Hence $g_{1}\left(u_{1}+u_{2}\right)=j \lambda=(j-i) \lambda+i \lambda \geq u_{1}+i \lambda=g_{1}\left(u_{1}\right)+g_{2}\left(u_{2}\right)$. Case 2: $M_{j}-\lambda \leq u_{1}+u_{2} \leq M_{j}$, for some $j \leq i$. As $u_{2} \geq M_{i}=M_{j}+\sum_{t=j+1}^{i} c_{\ell_{t}} \geq$ $M_{j}+(i-j) \lambda$, then $g_{1}\left(u_{1}+u_{2}\right)=u_{1}+u_{2}-M_{j}+j \lambda \geq u_{1}+(i-j) \lambda+j \lambda$ $=u_{1}+i \lambda=g_{1}\left(u_{1}\right)+g_{2}\left(u_{2}\right)$.

The case $u_{1}<0$ and $M_{i}-\lambda \leq u_{2} \leq M_{i}$ is similar to the previous one, so it will be omitted.

This function may differ from $f$ largely when $u<\gamma$. In such cases, and when $\gamma>\lambda$ we can use the following function, $g_{2}$, that provides a better approximation of $f$ for $u<0$ but differs from $f$ on the positive side.

$g_{2}(u)= \begin{cases}u+N_{r}+k c_{r}-(r+k) \lambda, & -\gamma-N_{r}-k c_{r} \leq u \leq-\gamma-N_{r}-k c_{r}+\lambda, k \geq 1, \\ -\gamma-(r+k) \lambda, & -\gamma-N_{r}-(k+1) c_{r}+\lambda \leq u \leq-\gamma-N_{r}-k c_{r}, k \geq 0, \\ u+N_{j}-j \lambda, & -\gamma-N_{j} \leq u \leq-\gamma-N_{j}+\lambda, j=1, \ldots, r, \\ -\gamma-j \lambda, & -\gamma-N_{j+1}+\lambda \leq u \leq-\gamma-N_{j}, j=0, \ldots, r-1, \\ u, & -\gamma \leq u \leq 0, \\ i \lambda, & i c_{1} \leq u \leq(i+1) c_{1}-\lambda, i \geq 0, \\ u-i c_{1}+i \lambda, & i c_{1}-\lambda \leq u \leq i c_{1}, i \geq 1,\end{cases}$ where $c_{1}=\max \left\{c_{j} \mid j \in \bar{S}^{+}\right\}$and $c_{r}=\min \left\{c_{j} \mid j \in \bar{S}^{+}\right\}$.

Proposition 11 Function $g_{2}$ is a valid superadditive lifting function for $f$ if $\gamma>\lambda$.

The proof of superadditivity of $g_{2}$ is similar to the proof of $g_{1}$, only many more cases need to be checked, thus we omitted it here.

\section{Computational experiments}

In this section we illustrate the use of the proposed inequalities to improve the integrality gap on a set of randomly generated instances. The conducted 
experimentes are preliminary, since it is outside of the scope of this paper to provide a deep study of the effectiveness of these inequalities in benchmark instances.

In order to test the impact of the inequalities developed for $X$, with different capacities, in the reduction of the integrality gap, we generate different sets of instances considering a maximization problem and compute, for each set, the average initial gap (IG), the average closed gap using inequalities (8) (CGS), and the average closed gap using the lifted inequalities (20) (CGL). Initial gaps are computed as $\frac{U B-O P T}{U B} \times 100$ where $O P T$ indicates the optimal value and $U B$ denotes the upper bound obtained by the linear relaxation of the problem. Moreover, closed gaps are calculated as $\frac{U B-I U B}{U B-O P T} \times 100$ where $I U B$ denotes the linear relaxation with inequalities (8) for CGS and, linear relaxation with inequalities (20) for CGL. For CGS, inequalities are added using the separation algorithm of Section 3 to the linear relaxation solution, and then the linear relaxation is solved again. The process is repeated until no new cuts are found. For inequalities (20) we use the same procedure while we only lift the inequalities (20). All computations are performed using the optimization software Xpress-Optimizer version 23.01.03 [8].

The test instances are generated randomly on the basis of the following data. We consider $n=50$, two possible values for $d$ (100 and 500), two possible values for the objective coefficient of $y$, denoted by $c_{y}$, and for each possible combination of $d$ and $\mathrm{co}_{y}$ we randomly generate the values of $c_{j}$ from three sets. For $d=100$ we consider two uniform distributions for intervals $I_{1}=[4,5]$, $I_{2}=[10,20]$, and another set $I_{3}=[4,6] \cup[9,11] \cup[14,16]$, where $c_{j}$ is assigned to each interval with probability $1 / 3$ and then it is generated using the uniform distribution for the corresponding interval. Similarly, for $d=500$ we consider $I_{4}=[15,17], I_{5}=[40,60], I_{6}=[10,30] \cup[40,60] \cup[70,90]$. These intervals allow us to test the cases where the coefficients are almost constant and the cases where coefficients belong to different magnitudes. Coefficients of $z_{j}$ are randomly generated in the interval $\left[\theta_{j}-20, \theta_{j}+20\right]$ where $\theta_{j}=-5 \mu_{j}$ and $\mu_{j}$ denotes the average value of the interval for $c_{j}$. Coefficient of $x_{j}$ is randomly generated in the interval $[10,15]$. For each possible combination of $d, c_{y}$ and interval for $c_{j}$, we generate 5 instances. In Table 1 we report the average results of those 5 instances.

Table 1 Average dual gaps and average closed dual gaps using inequalities.

\begin{tabular}{|c|c|c|c|c|c|c|c|c|c|}
\hline \multirow{2}{*}{$\mathrm{d}=100$} & \multicolumn{3}{|c|}{$I_{1}$} & \multicolumn{3}{c|}{$I_{2}$} & \multicolumn{3}{c|}{$I_{3}$} \\
\cline { 2 - 11 } & $I G$ & $C G S$ & $C G L$ & $I G$ & $C G S$ & $C G L$ & $I G$ & $C G S$ & $C G L$ \\
\hline \hline$c o_{y}=-10$ & 0.47 & 16.06 & 69.54 & 0.84 & 17.64 & 87.38 & 0.24 & 28.96 & 70.18 \\
\hline$c o_{y}=-1000$ & 68.79 & 87.5 & 93.16 & 50.95 & 55.17 & 93.57 & 53.81 & 90.66 & 94.45 \\
\hline \hline $\mathrm{d}=500$ & \multicolumn{3}{|c|}{$I_{4}$} & \multicolumn{3}{|c|}{$I_{5}$} \\
& $I G$ & $C G S$ & $C G L$ & $I G$ & $C G S$ & $C G L$ & $I G$ & $C G S$ & $C G L$ \\
\hline \hline$c o_{y}=-10$ & 0.36 & 3.31 & 39.04 & 0.62 & 14.92 & 56.55 & 0.35 & 21.70 & 56.25 \\
\hline$c o_{y}=-1000$ & 0.47 & 3.31 & 42.66 & 0.79 & 14.92 & 72.61 & 0.45 & 21.70 & 56.25 \\
\hline
\end{tabular}


It can be concluded from Table 1 that the improvement from use of the simple set-up flow covers (8), and the lifted inequalities (20) decreases as $d$ increases. Also lifting has a clear impact on the reduction of the initial gap in all tested cases. The impact of inequalities (8) depends on the coefficients $c_{j}$ considered. Besides, in most cases this impact is greater when the values of $c_{j}$ increase.

\section{Conclusions and future research}

In this paper we derive a family of valid inequalities, the set-up flow cover inequalities, for a feasible set $X$, which can be regarded as a variant of the SNFCN set where a new binary variable $y$, is associated with the capacity of the node. Based on these inequalities we provide a complete polyhedral characterization of the convex hull of $X$ when capacities on the arcs are constant. For the case of varying capacities, we lifted the set-up flow cover inequalities. The preliminary computational results were encouraging, suggesting further tests to study the effectiveness of these inequalities should be carried out in benchmark instances sets.

Future research directions include the investigation of the polyhedral structure in the case where $y$ is integer and the study of fast separation heuristics for the set-up flow cover inequalities. The main goal of this line of research is to apply the new inequalities to more general mixed integer problems such as lot-sizing, inventory-routing and network design problems.

Acknowledgements This work was supported by FEDER funds through COMPETE and by Portuguese funds through CIDMA and FCT within project PEst-C/MAT/UI4106/2011 with COMPETE number FCOMP-01-0124-FEDER- 022690.

\section{References}

1. Archetti, C., Bertazzi, L., Laporte, G., Speranza, M.G.: A Branch-and-Cut Algorithm for a Vendor-Managed Inventory-Routing Problem. Transportation Science 41, 382-391 (2007)

2. Balas, E.: Disjunctive programming: Properties of the convex hull of feasible points. Discrete Applied Mathematics 89, 3-44 (1998)

3. Christof, T., Löbel, A.: PORTA-POlyhedron Representation Transformation Algorithm. http : //typo.zib.de/opt-long projects/Software/Porta/(2008)

4. Correia, I., Gouveia, L., Saldanha-da-Gama, F.: Discretized Formulations for Capacitated Location Problems with Modular Distribution Costs. European Journal of Operational Research 204, 237-244 (2010)

5. Gu, Z., Nemhauser, G.L., Savelsbergh, W.P.: Lifted flow cover inequalities for mixed 0-1 integer programs. Mathematical Programming 85, 439-467 (1999)

6. Nemhauser, G.L., Wolsey, L.A.: Integer and Combinatorial Optimization, John Wiley \& Sons, New York (1988)

7. Padberg, M.W., Van Roy, T.J., Wolsey, L.A.: Valid Linear Inequalities for Fixed Charge Problems. Operations Research 33, 842-861 (1985)

8. Xpress-Optimizer. http : //www.fico.com/en/Products/DMTools/Pages/FICO Xpress - Optimization - Suite.aspx

9. Zhao, Y., Klabjan, D.: A Polyhedral Study of Lot-sizing with Supplier Selection. Discrete Optimization 9, 65-76 (2012) 